\title{
Project of the security portals for explosives detection using CEAS sensor
}

\author{
J. Wawer, Z. Bielecki, J. Mikolajczyk, J. Wojtas, R. Medrzycki, M. Nowakowski \\ Military University of Technology, \\ 2 Kaliskiego Str., 00-908 Warsaw, Poland
}

\section{Introduction}

Detection of any improvised explosive devices (IED) is an extremely important problem in the fight against terrorism, especially in the specific objects like airports. For this reason a broad range of countermeasures are needed. Practically explosives materials can be detected with particles of explosives, i.e. sniffers, and with bulk detection methods applying probing radiations (e.g. different imagine techniques). Both techniques use different signatures of explosives, which are presented to the system operator.

In the case of portals with sniffer methods (called trace portals), the air in the immediate vicinity of the controlled person is sampled and then analyzed [1]. During this procedure both objects contained explosives and just explosives particles could be detected. If any explosive is detected, the installed detection system is able to identify the specific signature and alert the operator. However, the location of the explosive material and its quantity is not known.

Portals applying probing radiations provide possibility to detect of an anomalies, caused by presence of explosives (called anomaly portals). The results are usually presented in visual way. These portals can only detect suspicious or dangerous objects such as weapons, packs, vests. In this technology, there are currently used a lot of instruments for example millimeter wave or terahertz cameras etc.

However, the anomaly portals are not able to identify explosives or other substances such as drugs. The decision of the detection of explosives devices mainly depends on the ability and skills of the operator. In contrast, the trace portals can be used to detect the presence or to identify the explosives basing on their vaporized particles and gases. Although there are other types of the portals, practically, the combination of these two technologies makes it possible to develop an effective tool for the detection of explosives devices. For this reason, currently available portals are equipped with complex analysis systems, which are able to detection of a wide range of specific materials. For example there are used magnetic metal sensors, the millimeter-wave detectors, the magnetometer, the electric field tomography, ion mass spectroscopy, etc. [2].

\section{Portal detection systems overview}

Commercial detection systems should be reliable and flexible [3]. Nowadays, one of the most sensitive detection methods is mass spectrometry (MS). The method offers higher sensitivity in comparison to other detection techniques. The main disadvantage of its is high price and design complexity. Over the past few years, the price of MS systems had significantly decreased and now they can be used in standard equipped portals. There are many companies that offer MS systems such as Syagen, Mass Spec Analytical [4,5].

Ion Mobility Spectroscopy (IMS) is conceptually similar to MS one. In relation to MS, the main advantage is that IMS does not use a vacuum system. Thus the size of the system, price and complexity are significantly reduced. However, the obtained accuracy is much lower. This is particularly important during detection of complex samples, where the test is performed for many compounds simultaneously. Such systems are manufactured by some companies: GE/lon Track, Smiths Detection, Sionek.

The IMS or MS detectors can be applied to gas chromatography (GC). The GC method provides high resolution (separation) of the compounds which could be detected by various techniques. The highest precision is achieved by using the MS detector. However, for applications such trace portals, there are applied a less expensive ones, such as electron capture (ECD), chemiluminescent (for $\mathrm{NO}_{2}$ group detection) or IMS. Such systems are manufactured by the Scintrex. 
Very promising and dynamically developing method is optical spectroscopy, especially visible (VIS), infrared (IR) and Raman spectroscopy. They can be used to identify the substances. However, the technology could not be so effective in the case of detection of complex compounds with very low concentration. At the same time it is also developed a millimeter-wave spectroscopy. It offers the possibility of explosives specification. The sensitivity of this method is not so high because of the small values of explosives absorption cross-section in the operated wavelength range.

For construction so-called hand-held devices very attractive are chemical sensors. In these systems, a matrix of detectors is very often used. The matrix may respond on detectable substance by changing the conductivity, wave propagation, fluorescence, or other markers. Chemical sensors are not very sensitive in the case of complex mixtures and may cause high levels of false alarms.

\section{Optoelectronic nitrogen dioxide sensor}

In the presented portal, an optoelectronic nitrogen dioxide sensor was applied. The sensor basis on the most sensitivity optoelectronic method called cavity enhanced absorption spectroscopy (CEAS). Because nitrogen dioxide $\left(\mathrm{NO}_{2}\right)$ is one of the nitrogen oxides, which often occurs in the explosives compositions, it also can be used as detection signature. In Fig. 1 chemical compositions of frequently used explosives are presented.

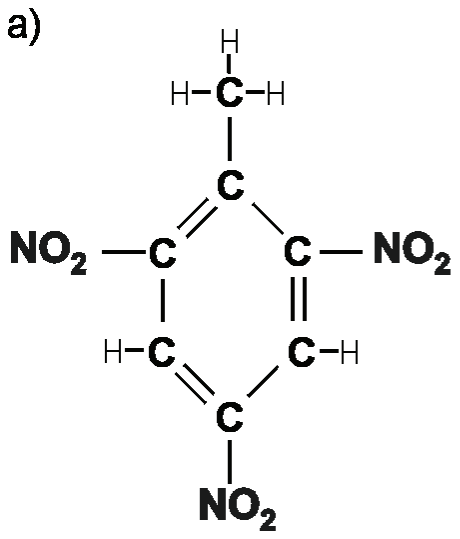

b)<smiles>O=[N+]([O-])N1CC2C1C[N+]([N+](=O)[O-])N2[N+](=O)[O-]</smiles>

Fig. 1.Chemical compositions: TNT (a), RDX (b)

The developed sensor makes it possible to detect trace concentrations $\mathrm{NO}_{2}$. CEAS is a modification of cavity ring down spectroscopy (CRDS) [6]. The sensitivity of CRDS sensors is higher, however in the practical implementation, such sensors requires efficient elimination of mechanical instabilities and the refraction coefficient variations. Moreover, to ensure appropriate storing of light in the resonator, the frequency of probing laser mode must be well matched to a longitudinal cavity mode. Therefore, when single mode lasers are used, a good electronic stabilization and synchronization of the cavity and the laser frequency are necessary.

In the CEAS, the off-axis arrangement of an optical cavity and laser beam is used. It eliminates optical feedback from the cavity to the light source (which is especially destructive for diode lasers stability). In the cavity, a dense structure of weak modes is obtained or the modes do not occur due to overlapping. Thanks to this, the CEAS system is much less sensitive to mechanical instabilities in comparison to CRDS one. CEAS sensors attain the detection limit of about $10^{-9} \mathrm{~cm}^{-1}$ [7]. Determination of tested gas concentrations basis on measurements of the optical radiation changes, which are caused by light absorption phenomenon. Therefore, both characteristic absorption spectrum of the investigated gas and proper selection of probing radiation source are very important. In the case of $\mathrm{NO}_{2}$, the maximum of the absorption spectrum is in the range of $400 \div 450 \mathrm{~nm}$ (Fig. 2). The absorption cross section $(\sigma)$ at these wavelengths exhibits several minima and maxima varying from about $3.5 \cdot 10^{-19}$ till $6 \cdot 10^{-19} \mathrm{~cm}^{2}[8]$. Moreover, there are no absorption interferences from other gases or vapors normally existing in the air. Thus, it could be assumed that the intensity changes of registered radiation are caused by changes of nitrogen dioxide concentration. The assumption does not take into consideration an influence of 
scattering by some aerosols and smokes existing in the air. However, the scattering effect can be minimized from the investigated gas using special filters.

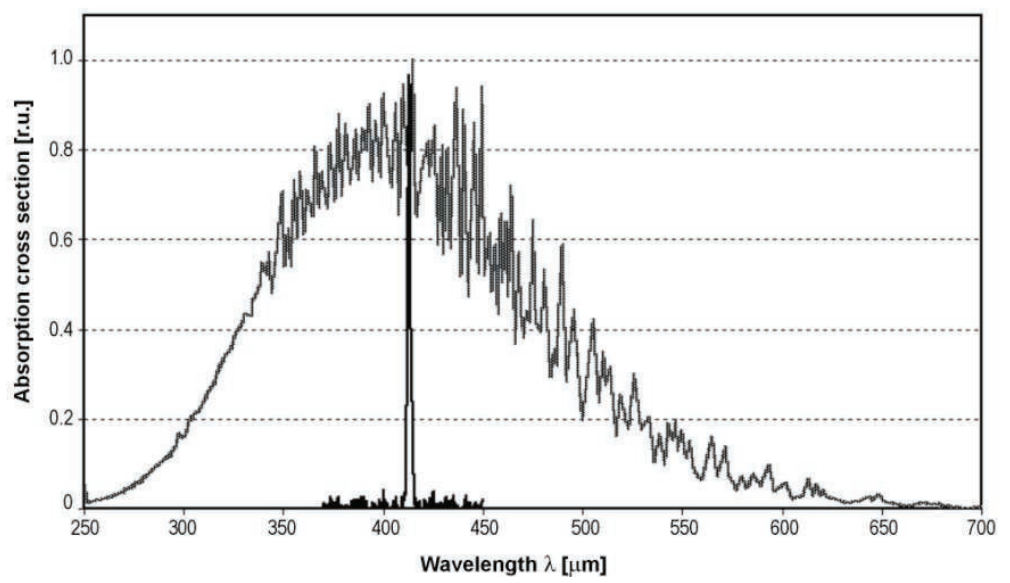

Fig. 2. Absorption cross-section of nitrogen dioxide and matched laser spectra the

The developed sensor consists of pulsed laser diode, diffraction grating and mirror, optical cavity, photomultiplier tube (PMT), analog to digital converter, and computer with special software (Fig. 3)

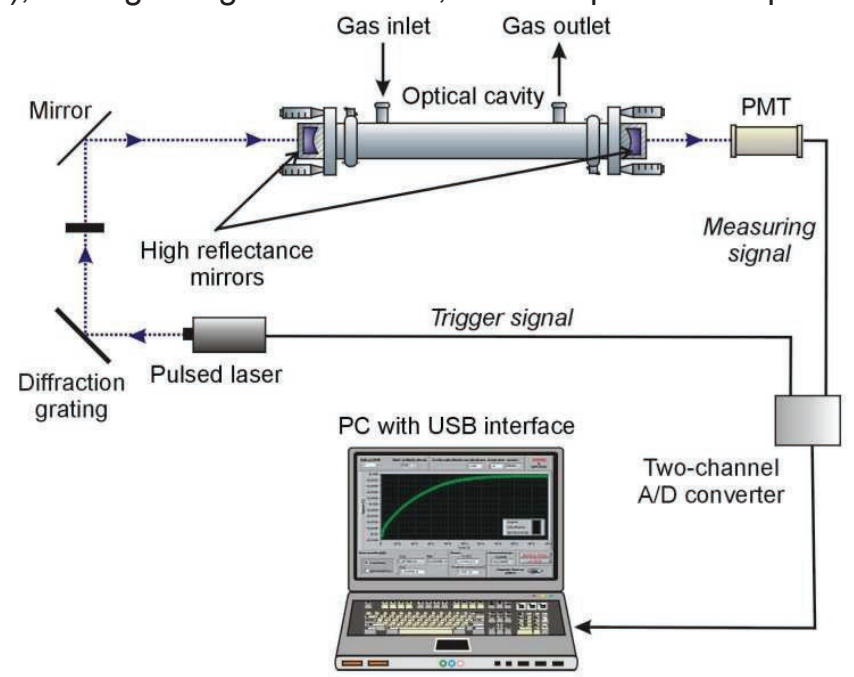

Fig. 3. Scheme of nitrogen dioxide sensor

In the sensor, there is used $500 \mathrm{~mW}$ pulsed diode laser (TopGaN) operating at the wavelength of $416 \mathrm{~nm}$. The laser generates pulses of radiation which FWHM duration time is about $50 \mathrm{~ns}$ and repetition rate about $1 \mathrm{kHz}$. Due to pulsed emission and broadband spectrum of the laser there was no problem of coupling with the cavity modes. The laser radiation is directed to the cavity using the diffraction grating and the mirror. The use of the diffraction grating eliminated the broadband fluorescence of the diode, which could be affected the output signal. The optical cavity is built of two mirrors which reflectivity reached value of 0.99995 at the wavelength of interest. The distance between the mirrors was $60 \mathrm{~cm}$. The output signal is registered with a photomultiplier tube (R7518, Hamamatsu). Next, the signal is digitized with fast 14-bit USB digital oscilloscope (CS328, Cleverscope) and measurement data are transmitted to the computer through USB interface. The $\mathrm{NO}_{2}$ concentration $(C)$ is determined with the special prepared software, using following formula

$$
C=\frac{1}{\sigma \cdot c}\left(\frac{1}{\tau}-\frac{1}{\tau_{0}}\right)
$$

where $\sigma$ - absorption cross section of the nitrogen dioxide, $c$ - light velocity, $\tau_{0}$ - decay time of the signal form the cavity, measured without absorber (nitrogen dioxide) inside the cavity, $\tau$ - decay time measured when cavity is filled with nitrogen dioxide [9]. 
Investigations of the developed $\mathrm{NO}_{2}$ sensor showed that its sensitivity reaches of $1 \mathrm{ppb}$ level and measurement uncertainty $10 \%$ above $10 \mathrm{ppb}$ can be ensured. Other parameters of the sensor are listed in Table 1.

Table. 1. $\mathrm{NO}_{2}$ optoelectronic sensor parameters

\begin{tabular}{|c|c|c|}
\hline No. & Parameter & Value \\
\hline 1. & Sensitivity $\left(\mathrm{NO}_{2}\right)$ & $1 \mathrm{ppb}$ \\
\hline 2. & Resolution of measurements & $0.2 \mathrm{ppb}$ \\
\hline 3. & Uncertainty $(\mathrm{C} \geq 10 \mathrm{ppb})$ & $10 \%$ \\
\hline 4. & Measurement time & $\leq 60 \mathrm{~s}$ \\
\hline 5. & Interface & USB 2.0 \\
\hline 6. & Dimensions (without notebook) & $80 \times 55 \times 30 \mathrm{~cm}$ \\
\hline 7. & $\begin{array}{c}\text { Weight (without notebook, and } \\
\text { accumulators) }\end{array}$ & $30 \mathrm{~kg}$ \\
\hline
\end{tabular}

\section{Security portal}

The preliminary research showed that the developed sensor $\mathrm{NO}_{2}$ can be effectively used to detect some explosives. Due to its functional virtues (power size, price, cost of operation and services) and parameters (sensitivity, measuring time), the sensor was applied to design the detection portal. The main elements of the designed portal are (Fig.4):

1. air supply system,

2. sampling system,

3. $\mathrm{NO}_{2}$ sensor.

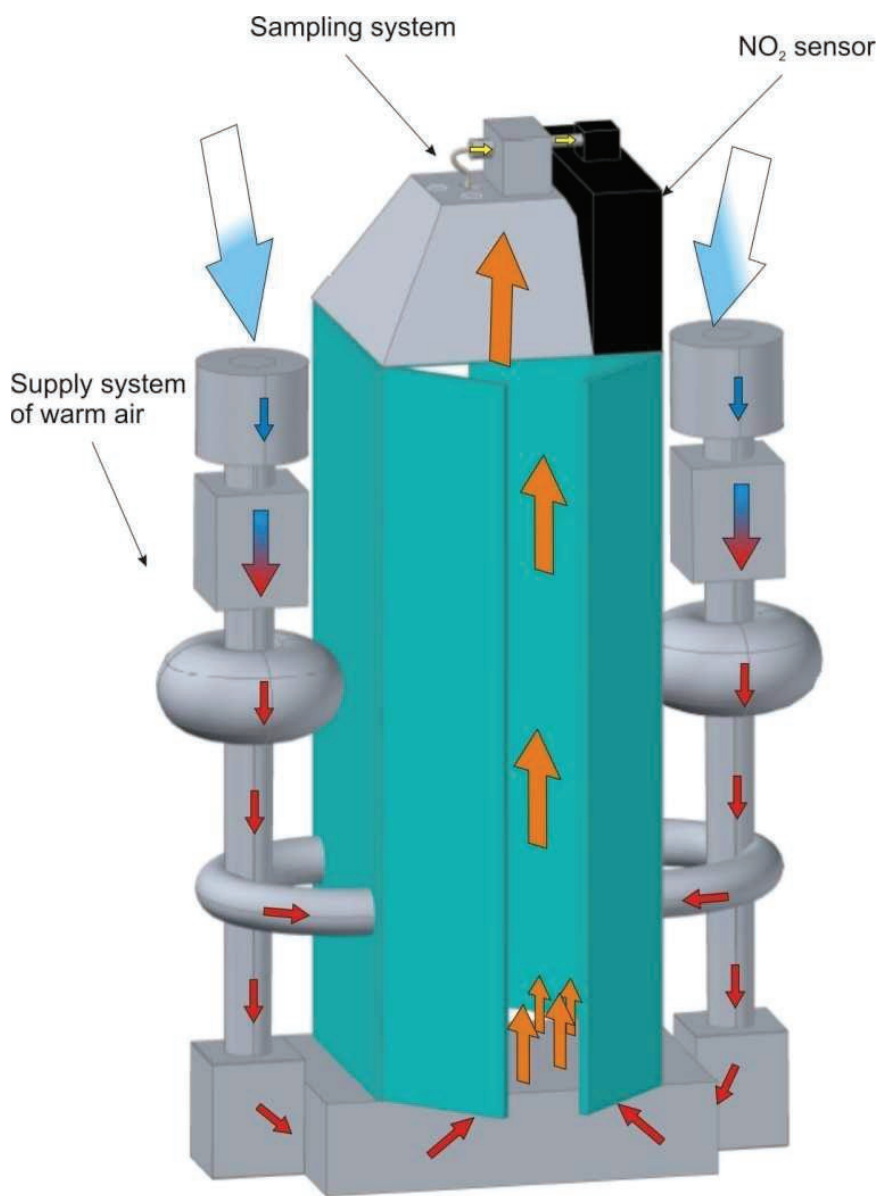

Fig. 4. The construction of the portal 
The main task of the air supply system is to collect of air samples from the portal (during person inspection) and to deliver them to the $\mathrm{NO}_{2}$ sensor. The system consists of two symmetrically positioned air turbines pumping the air into the portal. The probed air is filtered and heated up in the range of 30$40^{\circ} \mathrm{C}$. At the bottom of the portal, a layer made from filtering material is installed on (e.g. meets EU3 standard). It is used as another filtering stage and attenuator of air turbulence inside the portal as well. The air from the bottom uniformly flows through a filter at the rate dependent on the pressure difference in the portal. The rate is limited by the turbine speed at the range of $0.2-0.3 \mathrm{~m} / \mathrm{s}$. A very important element in the portal construction is the sampling system which is integrated with the air supply system. It is used to preparing specific air samples for the $\mathrm{NO}_{2}$ sensor. These air samples include explosives vapors. The sampling system is composed of moisture filter and filter for solid particles, the dimensions of which are larger than $5 \mu \mathrm{m}$. In such way, the samples delivered to the sensor are free from particles which could disturb of $\mathrm{NO}_{2}$ sensor operating. Moreover, the sensor is equipped with a unit capable of "cleaning" the cavity with an inert gas. This process is required for sensor calibration procedure.

People inspection is performed using an automated procedure of entrance monitoring and the trace dangerous particles detection. The block diagram of the procedures is presented in Fig. 5. Entrance monitoring is performed with electronically controlled door and video monitoring with use of specialized camera (SmartCamera, National Instruments). Thanks to signal from camera, it is possible to conduct an adequate preliminary analysis of the image, e.g. man behavior. An important element of the system is a gas flow control unit, which deliver portal air to the $\mathrm{NO}_{2}$ sensor. The sensor analyzing the air and sends alarm signals in case of trace dangerous particles detection.

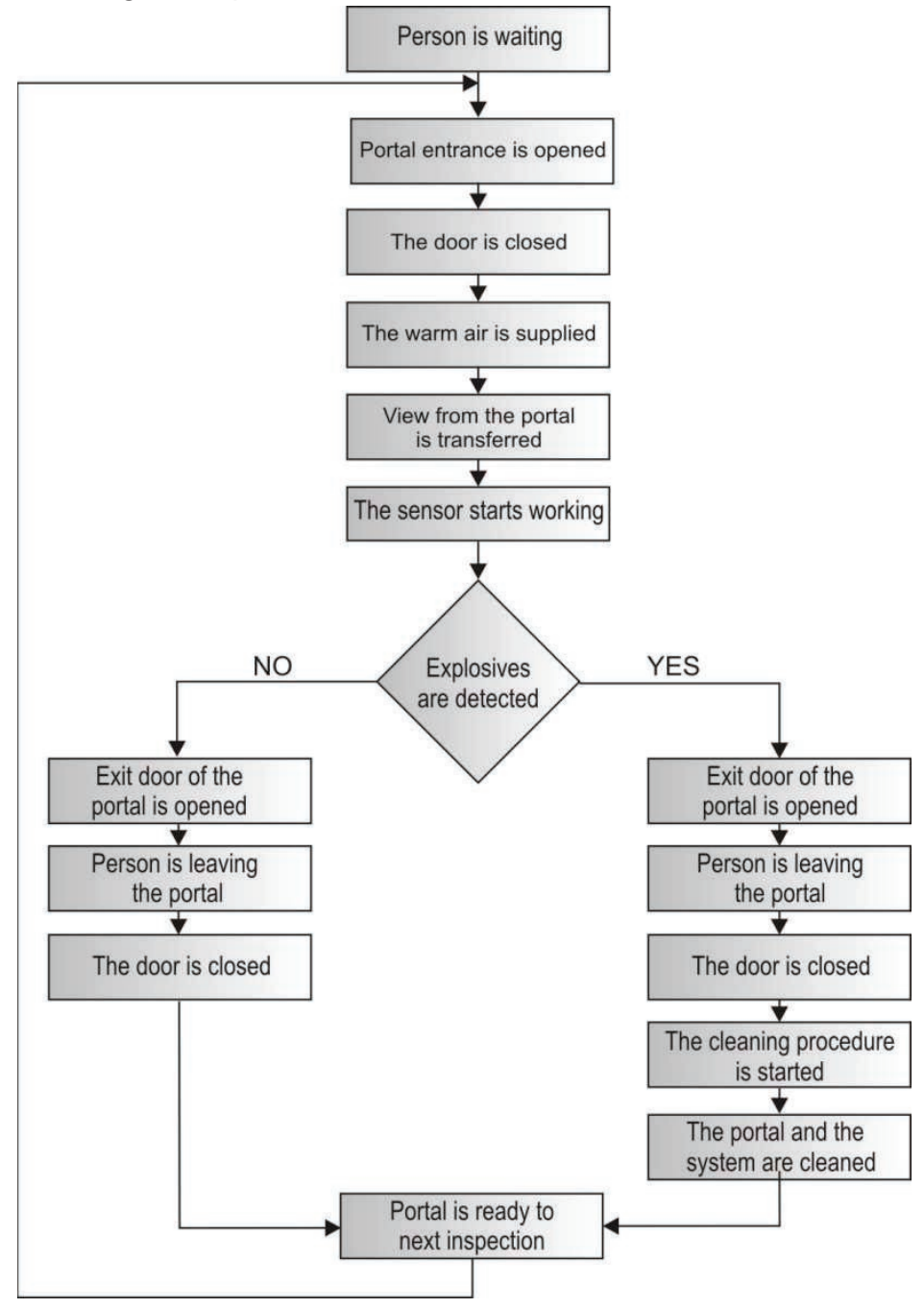

Fig. 5. Block diagram of the security portal automatic inspection 
From the sensor, the indication of a possible explosive is sent directly to the operator. At the same time the picture of the suspected person is taken. After inspection, an automatic procedure of cleaning system is started. During this procedure, the portal doors are closed and pure air flow is applied. In the sensor, elimination of the contaminated air is performed by controlled flow of inert gas. This procedure can also be applied to periodic monitoring of the sensor parameters (diagnostic procedure).

The appropriate time interval that is required to perform the detection procedures is preserved by the control entrance system. The inspected person has access to the portal in the case of readiness notification by the sensor. The portal is also equipped with the additional option of emergency access (manual mode).

\section{Summary and conclusions}

In the paper, the project and operation procedures of the walk-through trace detection portal designed to screen personnel for explosives was discussed.

For detection of explosives $\mathrm{NO}_{2}$ optoelectronic sensor was used. In the sensor CEAS technique was applied. Thanks to the photoreceiver and signal processing system optimization, the $\mathrm{NO}_{2}$ sensor sensitivity of $1 \mathrm{ppb}$ is achieved. Measurements with resolution of $0.2 \mathrm{ppb}$ and with uncertainty of $10 \%$ are possible. Special attention was paid to design security portal. The portal makes it possible to detect gas contamination in air flows over the body of the person being inspected. The portal was developed with the intent of producing a prototype system that could meet the requirements for airport screening of personnel.

\section{References}

[1] G. S. Settles, W. J. McGanna, Potential for portal detection of human chemical and biological contamination, SPIE Aerosense, Vol. 4378, 2001

[2] http://www.justnet.org/Lists/JUSTNET\%20Resources/Attachments/2129/00-portal\%20Primer_web.pdf

[3] R. L. Woodfin, Trace Chemical Sensing of Explosives, John Wiley \& Sons, Inc., 2007

[4] http://www.syagen.com/guardian-explosives-trace-portal-p-1-pc-1.html

[5] http://www.msaltd.co.uk/Detection-Equipment/Scentinel.aspx

[6] R. Engeln, G. Berden, R. Peeters, G. Meijer, Cavity enhanced absorption and cavity enhanced magnetic rotation spectroscopy, Review Of Scientific Instruments, Vol. 69, No. 11, pp. $3763-$ 3769, November, 1998

[7] J. Wojtas, A. Czyzewski, T. Stacewicz, Z. Bielecki, J. Mikolajczyk, „Cavity enhanced spectroscopy for NO2 detection", Proceedings of the SPIE, Volume 5954, pp. 174-178, 2005.

[8] J.Wojtas, A. Czyzewski, T. Stacewicz, Z. Bielecki, Sensitive detection of NO2 with Cavity Enhanced Spectroscopy, Optica Applicata, 36(4), pp. 461-467, 2006.

[9] J. Wojtas, Z. Bielecki, „Signal processing system in the cavity enhanced spectroscopy”, Opto-Electron. Rev. 16(4), pp. 44-51, 2008. 